\title{
Henri Mitterand - Critique génétique et sociocritique
}

Entretien avec Pierre-Marc de Biasi et Anne Herschberg Pierrot

Henri Mitterand, Pierre-Marc de Biasi et Anne Herschberg Pierrot

\section{(2) OpenEdition}

\section{Journals}

\section{Édition électronique}

URL : https://journals.openedition.org/genesis/121

DOI : $10.4000 /$ genesis. 121

ISSN : 2268-1590

\section{Éditeur :}

Presses universitaires de Paris Sorbonne (PUPS), Société internationale de génétique artistique littéraire et scientifique (SIGALES)

\section{Édition imprimée}

Date de publication : 20 juin 2010

Pagination : $59-63$

ISBN : 978-2-84050-697-3

ISSN : 1167-5101

\section{Référence électronique}

Henri Mitterand, Pierre-Marc de Biasi et Anne Herschberg Pierrot, « Henri Mitterand - Critique génétique et sociocritique », Genesis [En ligne], 30 | 2010, mis en ligne le 17 mai 2013, consulté le 30 mars 2023. URL : http://journals.openedition.org/genesis/121 ; DOl : https://doi.org/10.4000/genesis. 121 


\section{Critique génétique et sociocritique Entretien avec Pierre-Marc de Biasi et Anne Herschberg Pierrot}

Henri Mitterand

Henri Mitterand est l'un des fondateurs de la sociocritique en France1, et de la génétique zolienne. Il est aussi linguiste, sémioticien et stylisticien. Nous l'avons interrogé sur les relations complexes de la génétique et de la sociocritique.

\section{Sociocritique et sociogenèse}

PIERRE-MARC DE BIASI - Après une trentaine d'années de recul et d'expérience, quelle place donneriez-vous à la sociocritique et à la sociogenèse dans la construction $d u$ nouveau rapport critique qui s' est institué avec les manuscrits et la genèse des æeuvres? Au départ, la sociologie et l'analyse des manuscrits se présentaient plutôt comme deux approches assez éloignées, et vous avez fait partie de ceux qui ont montré qu'il ne s'agissait pas du tout de démarches incompatibles, situées aux antipodes l'une de l'autre, mais au contraire, d'univers intellectuels proches, et même solidaires.

HENRI MITTERAND - Effectivement, la sociocritique s'est d'abord construite sur une étude des textes achevés, des textes publiés. On a étudié ce qu'on pouvait imaginer du discours social inscrit dans la conscience de l'écrivain lorsqu'il écrit son roman : dans sa conscience ou dans son inconscient. Ce questionnement se situait sur le versant de la production. Mais on a étudié aussi l'autre versant : celui qui concerne la réception de l'œuvre par le lecteur, un lecteur lui-même habité par toutes sortes de stéréotypes, d'idées reçues, de présupposés, etc. Ces deux versants, on les envisageait effectivement à partir du texte achevé, c'està-dire qu'on allait chercher les traces d'une double productivité du système social dans l'œuvre publiée : non seulement du système objectif, mais aussi et surtout de la pensée du social, de la rêverie du social dans le discours de l'écrivain et dans le discours, également silencieux, du lecteur.
Ce qu'apporte la génétique, ce sont des traces objectives, matérielles, de ce travail intérieur et antérieur : la présence d'un monde de recherches, de réflexions, d'approximations, de tentatives de mise au net - et aussi d'illusions et de fantasmes.

P.-M. DE B. - Vous êtes même allé jusqu'à dire que l'épaisseur du manuscrit offre au chercheur un gisement plus large, plus profond, et finalement plus fructueux que le texte définitif pour rassembler les traces de ces impacts sociaux, de ces représentations sociales. Diriez-vous que la genèse permet d'identifier ces impacts comme les traces d' un projet délibéré de l'écrivain?

H. M. - Dans certains cas oui. Chez Zola, on voit bien qu'il y a une part de réflexion consciente, d'argumentation intérieure dans la rédaction des dossiers de travail. Philippe Hamon a bien montré la place du rhétorique dans les dossiers de travail de Zola ${ }^{2}$. Et dans le rhétorique, il y a le travail de l'inventio, qui se manifeste par un discours du sujet à la recherche de ses motifs et de sa diégèse. Aux premières lignes de l'Ébauche de Germinal, l'inventio apparaît dans une déclaration solennelle : « Je veux dépeindre le soulèvement des salariés contre le Capital. » On est bien ici au premier stade de la genèse, et on est également en présence d'un discours à caractère politicosocial affiché, ça ne fait pas de doute.

1. Avec Claude Duchet. Voir son entretien avec Anne Herschberg Pierrot et Jacques Neefs dans le numéro 6 de Genesis (1994).

2. Philippe Hamon (dir.), Le Signe et la consigne. Essai sur la genèse de l' $e u v r e$ en régime naturaliste. Zola, Genève, Droz, 2009. 
Simplement, on ne peut pas s'en tenir au repérage de ces certitudes, ou de ces hypothèses parfaitement explicites, sur le rapport entre la structure de la société, les systèmes idéologiques collectifs et individuels, et l'œuvre qui est en gestation. Il faut passer par-derrière... C'est tout l'enjeu d'une sociogenèse.

Anne Herschberg Pierrot - Vous voulez dire qu' en dehors des déclarations d'intention, l' univers génétique de l'avant-texte révèle cette dimension de l'insu idéologique, du stéréotype latent, etc., qui travaille l'écriture?

H. M. - Oui, il y a tout cela, bien entendu. La sociogenèse ne s'intéresse pas seulement à ce qu'il y a d'intentionnel et d'argumentatif dans les dossiers préparatoires ou dans l'œuvre : elle doit travailler aussi sur le narratif, le descriptif, le rêve, les métaphores, les recherches de phrasé. Cela dit, il me semble qu'il y aurait trois orientations possibles pour la génétique. Jusqu'à présent, on a essentiellement privilégié dans la critique génétique la reconstitution du jeu des avant-textes dans leur déroulement, les corrections qui les parsèment, etc., avec éventuellement des interprétations, mais qui repèrent plutôt les facteurs situés sur l'axe syntagmatique. Et l'on reste un peu au niveau de la structure superficielle. Il y aurait une autre orientation, complémentaire : une génétique, si j'ose dire, paradigmatique, qui essaierait de rechercher les structures profondes gouvernant l'émergence de la représentation et du texte ${ }^{3}$.

Je pense encore à une troisième orientation, qui serait celle d'une métagénétique, c'est-à-dire d'une réflexion qui élaborerait des problématiques pour la génétique, une critique de la génétique, essayant de cerner, de délimiter ses relations avec les autres techniques d'analyse des textes, sémiotique, sociocritique, thématique, psychanalyse.

P.-M. DE B. - C' est très compliqué, parce qu' avec les documents de genèse, ces méthodes sont confrontées à des processus très mouvants, temporalisés, conflictuels qui contredisent certains de leurs présupposés. Est-ce que le passage par les manuscrits n'a pas déjà conduit à problématiser sérieusement les outils d'analyse du texte, en disqualifiant, par exemple, ce que pouvait avoir de plus tranché, de plus immobile, ou de plus réducteur une certaine sociologie mécaniste de la littérature? Est-ce que la prise en considération des mouvements de l'écriture, des transformations du brouillon, n'est pas allée dans le sens d'une réflexion beaucoup plus fine sur l'idéologie dans ses rapports au texte et à la langue?

H.M. - Sûrement. D'ailleurs, ce qui a probablement manqué le plus à la sociocritique, c'est la combinaison des données du social et du linguistique (y compris dans sa dimension idiolectale). À la journée organisée par Gisèle Séginger sur Madame Bovary, je prenais l'exemple du paysage de Yonville, lors de la promenade de Rodolphe et d'Emma ${ }^{4}$. Quand on regarde bien ce paysage, Yonville vu d'en haut, on s'aperçoit que Flaubert travaille sur une gamme qui fait apparaître des métonymies, ou en tout cas des phénomènes de représentation en contiguïté (les nuages cédant la place à l'azur), des antonymies (l'azur, le ciel bleu, et les brouillards, les arbres qui percent l'azur, etc.), des hiérarchisations (hyponymes-hyperonymes, partie-tout, etc.), des métaphorisations. Il jongle avec un vocabulaire dont les lexies ou les lexèmes entrent virtuellement, et en langue, dans ce jeu de relations. Il joue d'un matériel associatif qui ne lui est pas propre, qui a servi à quantité de descriptions du même genre, de sorte que ce matériel finit par travailler tout seul... Le miracle c'est qu'ici le paysage n'est pas convenu, il est au contraire très singulier dans sa textualité et dans son effet de lecture. Il n'en provient pas moins d'une espèce d'autogenèse du texte, en raison même des relations internes de la langue. Il y a un ou deux mots qui sont les mots de base, le village, la colline, il y a une vision fondamentale, qui est la vision surplombante : à partir de là, le matériel se met en place, dans un travail de l'écriture qui transforme un certain degré d'universalité des données en absolue singularité. Et je ne parle pas du rythme, qui m'intéresse aussi beaucoup. Qui construira une sociocritique et une sociogenèse des accents et des cadences?

3. Voir ci-dessous l'article de Henri Mitterand sur le « Scénarique ». 4. Henri Mitterand, «La présence entêtée des signes », dans Gisèle Séginger (dir.), Madame Bovary et les savoirs, Paris, Presses de la Sorbonne Nouvelle, 2009. 
P.-M. DE B. - Ce que montre le brouillon, c' est la succession de deux moments assez distincts. Il y a d'abord un premier moment, où l'écrivain s'affronte beaucoup à la chose qu'il entend formuler. C' est une période où d'autres enjeux, d'ailleurs, que ce qu'il écrit, sont en cause : par exemple le cryptage, c'est-à-dire l'accumulation de signaux de références minimum (vis-à-vis de l'intertexte, de l' Histoire, de l'autobiographie, etc.) qui se trouvent inclus dans ces stratégies de métaphorisation, métonymie, etc. Et puis, après beaucoup de travail et d'efforts, advient un moment où le brouillon se met lui-même au poste de pilotage, un moment où quelque chose comme une sorte de systématicité de l'avant-texte s'étant mise en place, l' ouvre dépasse Flaubert et le prend par la main pour lui indiquer le chemin.

H. M. - Eh bien, justement, nous avons en ce domaine un travail extraordinaire à mener. Mais le matériel linguistique est déjà là, et il est social. Toutes ces analogies, ces métonymies, ces antonymies, ces hiérarchies, etc., sont fournies par la matière langagière du discours social. C'est bien la société qui est là-derrière. On découvre du discours social, qui est le discours du paysage tel qu'il s'est investi dans les habitudes de regard et d'expression de la collectivité, tel qu'il s'est investi aussi dans les descriptions les plus raffinées, comme «L'Azur » de Mallarmé, par exemple. Il y a des parentés intertextuelles qui montrent bien que le social est présent, qu'il émerge, non pour paralyser ou corseter le génie de l'artiste, mais pour le nourrir et étendre sa réserve d'accords.

\section{A. H. P. - Quel serait précisément l'intérêt d'aller voir les brouillons de ce passage?}

H. M. - Considérable. À un moment donné, Flaubert éprouve le besoin de situer ses deux personnages, c'est un problème d'artiste : comment donner au lecteur cette vision d'un petit village, comme ça, saisi d'en haut? C'est lui qui se pose le problème et, bien entendu, les dossiers apportent la trace des solutions qui se sont construites petit à petit. C'est fondamental : comment est-ce que ce propos, déjà antiquement codé, se retrouve complètement singularisé et rénové ?

P.-M. DE B. - On touche là les questions fondamentales de la sociologie dans l'analyse littéraire. Le brouillon est à la fois peut-être le meilleur écran de projection des grandes contradictions sociales et le médium le plus éclairant sur les grandes forces qui sont en acte dans l'écriture, mais il est quand même éminemment l' espace d' un acte individuel, et les outils de la sociologie ne sont pas toujours très bien adaptés pour saisir ce qui fait sa spécificité : le processus de pensée d'une personne.

H. M. - C'est pour cela qu'une sociocritique, et de même une analyse du discours littéraire, ne doivent jamais oublier la part de l'individuel, du rare, de l'exceptionnel.

\section{A. H. P. - Il faut passer par le stylistique, aussi?}

H.M. - Il faut passer par le stylistique, effectivement (c'està-dire par la conscience du style). J'ai un autre exemple, à propos de Madame Bovary, qui est peut-être de caractère plus socio-idéologico-critique : le dîner, à l'arrivée d'Emma et de Charles, dans l'auberge, avec Léon. J'ai essayé de regarder quels étaient tous les présupposés, tout le su de Flaubert, en ce qui concerne les manières - l'organisation d'un dîner, la place des convives, les propos échangés. Je me suis amusé à exploiter un peu Goffman. L'idée était de prendre ce texte comme un exemple de « mise en scène de la vie quotidienne », mais en saisissant tous les présupposés d'un savoir extraordinairement précis, fin, profond, sur les mœurs, et sur le langage des clichés. En suivant jusqu'au bout cette voie, on en viendrait peut-être à l'ethnographie ou à l'ethnocritique. Là il y a du réel à pleins bords, mais un réel nullement donné tel quel : transformé par le style, qui est, on le sait une affaire de vision - et une affaire de «poétique insciente».

Autre chose, à ce propos, en passant. Il manque un retour sur la sociologie marxisante. Est-ce qu'elle est morte ? Comment est-ce qu'on peut la faire revivre ? Je crois qu'on doit la faire revivre, mais avec quels instruments ? Sur la critique de la vie quotidienne, je pense aussi à Henri Lefebvre.

P.-M. DE B. - Et je le crois aussi. Est-ce que la chute du mur de Berlin a mis fin à la lutte des classes? Non. Marx est une valeur d' avenir. Les livres qu' il a écrits ou inspirés doivent rester ouverts, ils n'ont été qu'à moitié lus. Je pense surtout à la capacité que le marxisme nous donne de poser des questions radicales que d'autres systèmes ne 
nous permettent même pas d'imaginer. D'ailleurs, je me demande si la question du matérialisme en critique ne mériterait pas d'être reformulée - ne serait-ce que pour débusquer avec un peu plus de sagacité ce qui ressemble bien, aujourd' hui, à des retours de la transcendance, du providentialisme, ou de la téléologie dans la pensée de l'histoire littéraire.

H. M. - Oui, mais en ne négligeant pas le fait que - et c'est probablement ce qui manquait à la sociologie, à la sociocritique classique - la matière, c'est la langue. C'est-à-dire qu'on ne peut pas imaginer une critique matérialiste des textes sans s'intéresser au matériau premier qui est la langue. La langue, c'était étranger à des penseurs qui se situaient très au-dessus de tout ça. On ne se préoccupait pas beaucoup du signifiant. Un vrai matérialisme historique et dialectique en critique doit intégrer l'exploration de la langue. Je prenais l'exemple du paysage de Yonville : c'est de la langue, avant même, ou en tout cas en même temps que du regard.

P.-M. DE B. - Précisément. C'est dans le feuilletage des brouillons que s'observe le mieux cette langue en train de se mobiliser, de se configurer, de se restructurer. Et voilà ce qui pourrait offrir à l'analyse matérialiste de l'écriture une espèce de densité expérimentale sans précédent.

\section{Sociocritique, histoire littéraire et génétique culturelle}

P.-M. DE B.- J'aurai une question sur la manière dont la sociocritique à composante génétique se situe par rapport à cette histoire littéraire qui fait retour, d' une certaine façon, aujourd' hui. À l' intérieur de la sociologie, vous avez eu, avec Bourdieu, une volonté de saisir les æuvres à travers les antagonismes d'écoles, les cercles d'influences, les systèmes d'appartenances et d' exclusion, etc.

H. M. - Ceci relève de la sociologie de la littérature, de la sociologie des écrivains, des écoles, des rapports de champs. C'est aussi ce que fait très bien Alain Viala...

P.-M. DE B. - Mais pour regarder les brouillons, c' est un peu prendre la longue-vue à l' envers...
H. M. - Oui, là, on est loin des brouillons, on est loin de la matière singulière des grands textes.

A. H. P. - Quelles seraient alors les pistes à ouvrir dans ces relations entre génétique et histoire littéraire? Vous aviez évoqué l'idée d'une génétique culturelle, dont la vocation serait plus large que la génétique des textes et l'histoire littéraire. Est-ce toujours à l'ordre du jour, et qu' entendezvous exactement par là?

H. M. - Ah ! C'était la belle époque des grandes ambitions et d'un optimisme peut-être quelque peu mécaniste... Après ces grandes considérations générales sur la relation entre le roman zolien et les grands courants d'idées du $\mathrm{XIX}^{\mathrm{e}}$ siècle, je suis plutôt revenu, aujourd'hui, à une espèce de consommation gourmande des œuvres. J'en suis peutêtre revenu à des points de vue esthétisants ! Pour résister, en somme, à cette mécanisation des études qui au fond considère que n'importe quel texte de la production littéraire mérite d'être étudié, commenté ; et aussi résister à la dictature de l'analyse argumentative dans les préoccupations analytiques ou pédagogiques. Ceci n'est qu'une parenthèse.

En revanche, oui, si on multiplie les analyses concrètes, je pense que l'on finira par arriver à ce genre de synthèse, à une génétique culturelle capable de répondre à de vastes questions. Par exemple : comment situer le travail de Zola écrivant Germinal au sein du grand mouvement de fantasmes, d'idées, d'inquiétudes, etc., qui a accompagné la renaissance du mouvement ouvrier?

P.-M. DE B. - Mais pour y parvenir, il faudra imaginer la refonte génétique de notions comme celles de source, de documentation ou d'intertexte. Une refonte qui permette de comprendre qu'il y a un rapport fort entre la lecture, la note, l'écriture, la rature, la sélection, le recyclage, le jeu avec les savoirs. Je pense à l' histoire des religions, par exemple, chez Flaubert : à cette connaissance de la mythographie allemande qui nourrit son écriture, au ressourcement de sa pensée à travers la Revue des Deux Mondes. Comment penser la culture d'un écrivain, la confluence des cultures qui se croisent en lui ? Il y a là une vieille ambition de la sociologie de la littérature, l'étude des transferts culturels, qui trouverait dans l'univers de 
l'avant-texte une voie royale pour donner forme à ce que vous appelez la génétique culturelle. Qu'est-ce que vous en pensez?

H. M. - Absolument d'accord. Cela suppose une érudition, une culture de l'analyste, une connaissance de tous les textes que l'écrivain a lui-même avalés, mais également des milieux qu'il a fréquentés - et aussi un sens des bonnes questions, de perception des perspectives...

A. H. P. - Je voudrais revenir sur les dossiers de genèse : est-ce qu'il y a des types de questions qui concernent la sociogenèse ou la sociocritique et qui sont relatives aux différentes étapes du travail de l'écrivain ? Est-ce qu' il y a des types de questions que la sociogenèse poserait plus volontiers aux scénarios, aux brouillons?

H. M. - La question de la logique, peut-être. Dans la construction d'un scénario, en tout cas, quand il s'agit de Zola - je ne peux pas parler d'un autre - on voit bien qu'il y a une logique sous-jacente, il y a une pensée de la succession des causes et des conséquences. Tel personnage, dans telle situation, peut ou ne peut pas faire tel acte. Il entre dans un double réseau de contraintes et de virtualités : celles, internes, des déterminations qui lui ont été attribuées au départ de l'ébauche (il est ouvrier, ou homme d'affaires, ou théâtreux, etc.) ; et celles, externes, du capital culturel, et intertextuel, investi dans l'œuvre.

P.-M. DE B. - Mais, en même temps, dans l'écriture de Zola, ce qui nous frappe le plus aujourd' hui, ce qui nous enchante, c'est qu'après cette rationalisation préliminaire, dans la rédaction, des télescopages fous ont lieu entre des lignes causales tout à fait indépendantes, et que ces télescopages produisent des images, des rêveries, des représentations oniriques ou des situations symboliques que rien ne laissait attendre : une écriture du fantasme qui complexifie à l' extrême la logique initiale des causes et des conséquences, parfois jusqu'à la renverser.

H.M. - Oui, tout à fait. J'ai souvent évoqué un certain degré de délire à son propos, et, partant, la nécessité de le dé-lire, de le lire avec d'autres lunettes que les siennes propres. C'est là qu'est la difficulté, et le cas de Zola n'est toujours pas réglé. Les malentendus ont la vie dure : on continue à débiter des généralités ou des observations critiques accrochées à ce vieux mot de «naturalisme », qu'il a popularisé un jour pour son malheur. 